\title{
Multi-objective Optimisation in Inventory Planning with Supplier Selection
}

\author{
Seda Türk, Ender Özcan, Robert John \\ ASAP Research Group, School of Computer Science, University of Nottingham, NG8 1BB, \\ Nottingham, UK
}

\begin{abstract}
Supplier selection and inventory planning are critical and challenging tasks in Supply Chain Management. There are many studies on both topics and many solution techniques have been proposed dealing with each problem separately. In this study, we present a two-stage integrated approach to the supplier selection and inventory planning. In the first stage, suppliers are ranked based on various criteria, including cost, delivery, service and product quality using Interval Type-2 Fuzzy Sets (IT2FS)s. In the following stage, an inventory model is created. Then, an Multi-objective Evolutionary Algorithm (MOEA) is utilised simultaneously minimising the conflicting objectives of supply chain operation cost and supplier risk. We evaluated the performance of three MOEAs with tuned parameter settings, namely NSGA-II, SPEA2 and IBEA on a total of twenty four synthetic and real world problem instances. The empirical results show that in the overall, NSGA-II is the best performing MOEA producing high quality trade-off solutions to the integrated problem of supplier selection and inventory planning.

Keywords: Interval type-2 fuzzy, Evolutionary computation, Metaheuristic, Optimisation.
\end{abstract}

Email addresses: syt@cs.nott.ac.uk (Seda Türk), exo@cs.nott.ac.uk (Ender Özcan), rij@cs.nott.ac.uk (Robert John) 


\section{Introduction}

In today's competitive and connected environment, many commercial organisations value effective management of the flow of materials considering the relationships between vendors, manufacturers, distribution centres, customers and other services for success (Thomas \& Griffin, 1996). The integration of all facilities which add value for buyers from the procurement of raw materials to the distribution of end products can be broadly defined as Supply Chain Management(SCM) (Thomas \& Griffin, 1996, Setak et al., 2012). In SCM, it is crucial to be working with dependable suppliers and planning the inventory via efficient allocation of the resources in the supply chain for a competitive advantage Vonderembse \& Tracey (1999). Choosing a supplier can impact on the cost and the quality of products. Evaluation and selection of suppliers is a critical issue in a supply chain. On the other hand, inventory planning is an integrated process handling the inventory across the entire network from suppliers to customers. Miller et al. (2011); Miller \& John (2010) pointed out that the supply chains with well managed inventory considers satisfying the demand, preventing stock outs and reducing holding costs - where stock is kept in the store for an undesirable period of time.

In most of the previous work, supplier selection and inventory planning are treated as separate problems. Moreover, many previous studies on supplier selection focus on fuzzy systems formulating the problem as a Multiple Attribute Decision Making (MADM) problem and taking the requirements of decision makers into account as well during the solution process. For example Chen et al. (2006); Pattnaik (2011); A.Sarkar \& Mohapatra 2006) and Gong (2013) studied an MADM approach based on Type-1 Fuzzy Sets and Interval Type-2 Fuzzy Sets (IT2FS), respectively. The supplier selection problem can be formulated as a multi-objective problem and so some of the previous work investigated multi-objective models looking into the trade-off between minimisation of total cost and lead time (Mastrocinque et al. 2013), minimisation of total cost and maximisation of customer service quality (Liao et al., 2011), minimising total 
cost, maximisation of customer service quality and capacity (Altiparmak et al. 2006). A thorough review of previous studies on supplier selection, covering a variety of exact and (meta)heuristic methods as well as computational intelligence techniques, can be found in (Ho et al., 2010). In this study, we extend the work of Ordoobadi (2009) in which Type-1 Fuzzy Sets are used to capture the uncertainty in the decision making process and (Turk et al. 2014) in which it has been observed that IT2FSs can deal with the linguistic uncertainty better. Hence, we utilise an Interval Type-2 Fuzzy System for evaluating suppliers.

Inventory management is an integrated process handling the inventory across the entire network from suppliers to customers. Miller et al. (2011); Miller \& John (2010) pointed out that the supply chains with well managed inventory considers satisfying the demand, preventing stock outs and reducing holding costs - where stock is kept in the store for an undesirable period of time. In order to solve inventory planning problem individually, a range of methods have been used, including genetic algorithms (Rezaei \& Davoodi, 2008), multiobjective algorithms (Liao et al., 2011; Shankar et al., 2013, Zhang et al., 2016) and hybrid approaches (Mahnam et al. 2009).

Supplier selection combined with effective inventory planning has been researched by a number of researches (Ghodsypour \& Brien, 2001, Mohammaditabar \& Ghodsypour, 2014; Parhizkari et al. 2013). Majority of the previous work treated SCM problems as a single objective problem. Although some studies considered multiple objectives, the problem was dealt with using an approach designed for optimising a single objective which was obtained by crashing multiple objectives into one via some scalarisation method, such as, weighted sum. However, the weakness of such approaches arises mainly due to their performance sensitivity to varying weights and their inability to obtain multiple trade-off solutions simultaneously after a run (Kim \& de Weck, 2006). Therefore, in our study, three Multi-objective Evolutionary Algorithms (MOEA)s are used: NSGA-II, SPEA2 and IBEA. In addition, previously proposed heuristic optimisation approaches could suffer from premature convergence (Esmin et al. 2015). In this study, two algorithms, namely NSGA-II and SPEA2 containing 
a diversity mechanism against this issue are utilised. The third MOEA used in our study, that is IBEA does not necessitate a diversity preservation method as the indicators such as hypervolume reportedly performs well across a wide range of problems (Zitzler \& Künzli, 2004). Moreover, as far as we know, in none of the previous work covered above, the parameter settings of search methods were tuned. Nevertheless, evolutionary algorithms like any other stochastic local search method require setting of algorithmic parameters and selected setting could have an essential impact on their performance. Identifying the optimal/best parameter settings for a search algorithm is one of the challenging tasks in designing an effective solution to the problem in hand (Deb, 2007). However, in our study, parameters of three evolutionary algorithms are tuned.

Only a few previous studies explored multi-objective supplier selection informed inventory planning. In this study, we investigate a two-stage integrated solution approach to solve the integrated supply chain problem of supplier selection and inventory planning. The first component involves solving the supplier selection problem using an IT2FS. Following that an inventory model is developed to investigate how supplier risk affects cost. The parameters of three MOEAs are first calibrated using the Taguchi approach (Taguchi \& Yokoyama, 1993). After the tuning parameters of these algorithms, each algorithm with the best setting is applied to the problem in order to examine the trade-off between operational cost of a supply chain and risk of suppliers. The performances of NSGA-II, SPEA2 and IBEA are evaluated using well known metrics on twenty four different problem instances with different characteristics and sizes, where four of them are real world problem instances and twenty of them are randomly generated based on those instances.

This paper is organised as follows. Section 2 introduces Type-2 Fuzzy Logic (T2FL) and multi-objective optimisation including MOEAs and performance metrics commonly used in the area. Section 3 provides the problem explanation and the proposed two-stage solution approach. Section 5 presents the experimental design and computational results. Section 6 discusses the conclusions and future work. 


\section{Background}

This section introduces the background for the techniques used as a part of the proposed approach and provides an overview of related studies in the scientific literature.

\subsection{Type-2 Fuzzy Logic}

In many problems, knowledge comprises of objective knowledge which is the formal description of the problem, i.e., mathematical model and subjective knowledge which encapsulates the linguistic information. Generally in mathematical models, subjective knowledge is overlooked (Ross, 2004). However, Zadeh (1965) overcame this issue by introducing fuzzy sets. Mendel \& John (2002) provided the following list of situations where T1FS could be insufficient for capturing the uncertainty in the problems:

1. Meaning of a word often relates to perception and so it could vary from one person to another with the perception.

2. Further uncertainty may arise if a group of experts do not agree on the definition of the consequents of a fuzzy system.

3. The input activating a T1FL system may be noisy, and therefore imprecise.

4. The data used for parameter tuning of a T1FL system could be noisy.

Nevertheless, the 3 dimensional fuzzy sets generated using Type-2 Fuzzy Sets (T2FS)s are extremely complicated, hence it can not be easily understood and applied. Because of this complexity, many T2FSs applications have been modelled using Interval Type-2 Fuzzy Logic Systems (Greenfield et al., 2012). The difference between T2FSs and IT2FSs is that for IT2FS, the membership function is an interval. This allows us to cope with uncertainty associated with the membership grades. We use IT2FS to depict the ambiguity inherent in the supplier selection problem.

\subsubsection{Basic Concepts of IT2FS}

In this section, we provide the fundamentals of IT2FS as explained in Mendel et al. (2006). 
Definition 2.1. (Mendel et al., 2006) In the universe of discourse $X$, a Type-2 Fuzzy Set $\tilde{A}$ can be assigned by a Type- 2 membership function $\mu_{\tilde{A}}$ indicated as:

$$
\tilde{A}=\left((x, u), \mu_{\tilde{A}}(x, u)\right) \mid \forall x \in X, \forall u \in J_{x} \subseteq[0,1]
$$

where $x \in X$ and $u \in J_{x} \subseteq[0,1]$ in which $0 \leq \mu_{\tilde{A}}(x, u) \leq 1$. The primary membership function is depicted as $J_{x} \subseteq[0,1]$. It is also demonstrated as:

$$
\tilde{A}=\int_{x \in X} \int_{u \in J_{x}} \mu_{\tilde{A}}(x, u) /(x, u) J_{x} \subseteq[0,1]
$$

where $\iint$ denotes a union over all admissible $x$ and $u$.

Definition 2.2. (Mendel et al. 2006) $\tilde{A}$ is defined as a Type-2 Fuzzy Set in the universe of discourse $X$ expressed by the Type- 2 membership function $\mu_{\tilde{A}}$. When all $\mu_{\tilde{A}}(x, u)=1$ for $\forall x \in X$ and $u \in J_{x} \subseteq[0,1], \tilde{A}$ is termed an Interval Type-2 Fuzzy Set depicted as:

$$
\tilde{A}=\int_{x \in X} \int_{u \in J_{x}} 1 /(x, u) J_{x} \subseteq[0,1]
$$

where $J_{x} \subseteq[0,1]$, i.e.

Definition 2.3. (Mendel et al. 2006) The IT2FS can be considered as a particular case of type 2 fuzzy set, where the upper and lower membership functions are both Type- 1 membership functions, respectively. As an example, a trapezoidal IT2FS $\tilde{A}_{i}$ for all $x \in X$ represented by;

$$
\begin{array}{r}
\tilde{A}_{i}=\left(\tilde{A}_{i}^{U}, \tilde{A}_{i}^{L}\right)=( \\
\left(a_{i 1}^{u}, a_{i 2}^{u}, a_{i 3}^{u}, a_{i 4}^{u} ; h_{1}\left(\tilde{A}_{i}^{U}\right), h_{2}\left(\tilde{A}_{i}^{U}\right)\right), \\
\left(a_{i 1}^{l}, a_{i 2}^{l}, a_{i 3}^{l}, a_{i 4}^{l} ; h_{1}\left(\tilde{A}_{i}^{L}\right), h_{2}\left(\tilde{A}_{i}^{L}\right)\right)
\end{array}
$$

where $h_{j}\left(\tilde{A}_{i}^{U}\right)$ and $h_{j}\left(\tilde{A}_{i}^{L}\right)$ for $1 \leq j \leq 2$ depict membership values of the corresponding elements $a_{i(j+1)}^{u}$ and $a_{i(j+1)}^{l}$, respectively (Hu et al. 2013). The height of each constituent membership function is not explicitly defined as it is assumed to be equal to 1 . 

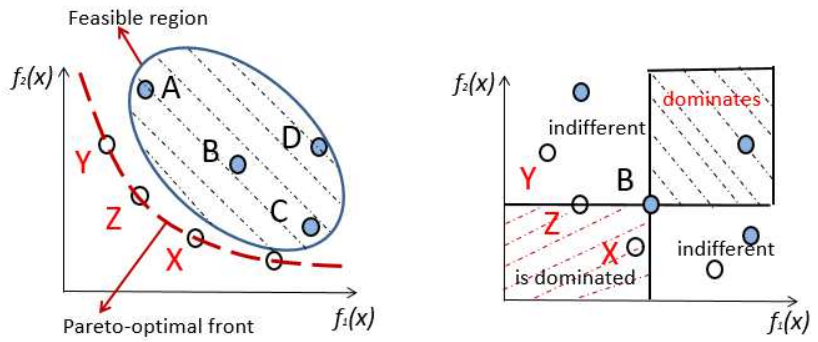

Figure 1: Pareto optimality in objective space(left) and the possible relations of solutions in objective space(right) (Zitzler, 1999).

\subsection{Multi-Objective Optimisation}

Although, a single objective optimisation technique performs a search to obtain a single solution to a given problem instance, a multi-objective optimisation is concerned with simultaneous minimisation and/or maximisation of multiple objectives yielding a set of trade-off solutions which are broadly equivalent (Zit-) zler, 1999).

\subsubsection{Basic Concepts of Multi-Objective Optimisation}

Generally, multi-objective problems include $n$ decision variables, $m$ objective functions and $j$ constraints. Objective functions and constraints are functions of the decision variables indicated as follows;

$$
\begin{array}{lr}
\text { minimise } & \boldsymbol{f ( x ) =}\left(f_{1}(x), f_{2}(x), \ldots, f_{m}(x)\right) \\
\text { subject to: } & \boldsymbol{e}(\boldsymbol{x})=\left(e_{1}(x), e_{2}(x), \ldots, e_{j}(x)\right) \leq 0 \\
\text { where } & \boldsymbol{x}=\left(x_{1}, x_{2}, \ldots x_{n}\right) \in \mathrm{X}, \\
& \boldsymbol{y}=\left(y_{1}, y_{2}, \ldots, y_{m}\right) \in \mathrm{Y}
\end{array}
$$

where $\boldsymbol{x}$ represents decision vector while $\boldsymbol{y}$ is objective vector in X decision space and Y objective space. Condition of $\boldsymbol{e}(\boldsymbol{x}) \leq 0$ provides the set of feasible solutions (Zitzler, 1999). 
Definition 2.4. Feasible Set (Zitzler, 1999); The set of decision vectors $\boldsymbol{x}$ which satisfies constraints $\boldsymbol{e}(\boldsymbol{x})$ is named as the feasible set $\boldsymbol{F} \boldsymbol{S}(\boldsymbol{x})$ :

$$
\boldsymbol{F S}(\boldsymbol{x})=\{x \in X \mid e(x) \leq 0\}
$$

In a single objective optimisation problem, the feasible set is totally ordered according to objective function $\boldsymbol{f}(\boldsymbol{x})$ : for two solutions $x_{1}, x_{2} \in \boldsymbol{F} \boldsymbol{S}(\boldsymbol{x})$ either $f_{1}(x) \geq f_{2}(x)$ or $f_{1}(x) \leq f_{2}(x)$. The aim is to find $\boldsymbol{f}(\boldsymbol{x})$ with maximum value. Nevertheless, when a number of objectives is considered, the feasible set is partially ordered (Zitzler, 1999).

As an example, in Figure 1, where $f_{1}(x)$ represents risk, $f_{2}(x)$ denotes cost. In this example, two objectives generally conflict with each other: high risk increases the cost while low risk decreases the cost. In Figure 1 on the left, there are several points as solutions; A, B, C. The solution $\mathrm{Z}$ is better than the solution $\mathrm{B}$ with higher performance and lower cost. On the other hand, the solution $\mathrm{Y}$ is better than solution A. Therefore, decision maker can choose an appropriate solution from the "equivalent" trade-off solutions (Zitzler, 1999).

Definition 2.5. Pareto Dominance (Zitzler, 1999); For any two decision vectors $x_{1}$ and $x_{2}$;

$$
\begin{aligned}
& x_{1} \succ x_{2} \text { if } f_{1}(x)>f_{2}(x) \\
& x_{1} \succeq x_{2} \text { if } f_{1}(x) \geq f_{2}(x) \\
& x_{1} \sim x_{2} \text { if } f_{1}(x) \nsupseteq f_{2}(x) \wedge f_{2}(x) \ngtr f_{1}(x)
\end{aligned}
$$

where $x_{1} \succ x_{2}, \quad x_{1} \succeq x_{2}$ and $x_{1} \sim x_{2}$ represent ' $x_{1}$ dominates $x_{2}$ ', ' $x_{1}$ weakly dominates $x_{2}$ ' and ' $x_{1}$ is indifferent to $x_{2}$ ' in a sequence. In Figure 1 on the right, the light grey rectangle area shows the region in objective space dominated by the solution B while the dark grey rectangles represent the areas which contain the solution vectors dominating the solution B. All solutions in the remaining region of the objective space are indifferent to the solution B as expressed in the last line of Equation 7

Definition 2.6. Pareto Optimality (Zitzler, 1999); A decision vector $\boldsymbol{x} \in$ 
$\boldsymbol{F S}(\boldsymbol{x})$ is nondominated considering a set $\boldsymbol{P S}(\boldsymbol{x}) \subseteq \boldsymbol{F S}(\boldsymbol{x})$ if;

$$
\forall x_{f s} \in \boldsymbol{F S}(\boldsymbol{x}): x_{f s} \succ \boldsymbol{x}
$$

All pareto-optimal solutions are referred to as pareto-optimal set where the corresponding objective vectors form the pareto-optimal front or surface. In Figure 1, white points demonstrate pareto-optimal solutions: there is no single optimal solution, but a set of optimal trade-off solutions.

Definition 2.7. Non-dominated Sets and Fronts (Zitzler, 1999);

Let $\boldsymbol{P S}(\boldsymbol{x}) \subseteq \boldsymbol{F S}(\boldsymbol{x})$, the function $\boldsymbol{p}(\boldsymbol{P S}(\boldsymbol{x}))$ gives the set of non-dominated decisions vectors in $\boldsymbol{P S}(\boldsymbol{x})$.

$$
\boldsymbol{p}(\boldsymbol{P S}(\boldsymbol{x}))=\left\{x_{f s} \in \boldsymbol{F} \boldsymbol{S}(\boldsymbol{x}) \mid x_{f s} \text { is non-dominated considering } \boldsymbol{F} \boldsymbol{S}(\boldsymbol{x})\right\}
$$

The set $\boldsymbol{p}(\boldsymbol{P S}(\boldsymbol{x}))$ is the non-dominated set in $\boldsymbol{F} \boldsymbol{S}(\boldsymbol{x})$, the corresponding set of objective vectors $\boldsymbol{f}(\boldsymbol{p}(\boldsymbol{P} \boldsymbol{S}(\boldsymbol{x})))$ is non-dominated front with respect to $\boldsymbol{F S}(\boldsymbol{x})$. In addition, the set $\boldsymbol{X} \boldsymbol{p}=\boldsymbol{p}(\boldsymbol{F S}(\boldsymbol{x}))$ is namely the pareto-optimal set and the set $\boldsymbol{Y} \boldsymbol{p}=\boldsymbol{f}(\boldsymbol{X} \boldsymbol{p})$ is called as the pareto-optimal front.

\subsection{Multi-objective Evolutionary Algorithms}

As the goal in multi-objective optimisation based on a metaheuristic is to obtain a set of trade-off solutions at the end of the search process for the decision makers, population based search techniques (which use multiple solutions during the search), in particular multi-objective evolutionary algorithms (MOEAs) are naturally preferred. A variety of MOEAs with differing algorithmic components, such as diversity maintenance, replacement, have been previously proposed and more can be found in Zitzler \& Thiele (1999); Zitzler et al. (2000); Konak et al. (2006). This work considers three MOEAs: Non-dominated Sorting Genetic Algorithm II (NSGA-II) (Deb et al., 2002), Strength Pareto Evolutionary Algorithm 2 (SPEA2) (Zitzler et al., 2002), Indicator-Based Evolutionary Algorithm (IBEA) (Zitzler \& Künzli, 2004).

NSGA-II is an elitist MOEA based on a non-dominated sorting method. In order to provide a better spread of solutions and convergence to pareto-optimal 
solutions, NSGA-II uses a crowding distance approach to sort individuals (Deb et al. 2002). Initially, a population $P_{1}$ of size $N$ is randomly generated and then those $N$ individuals are sorted into different non-domination levels. Then, an offspring population $Q_{1}$ of size $N$ is created using the individuals in $P_{1}$ and applying crossover and mutation operators with associated probabilities (rates). $P_{1}$ and $Q_{1}$ are merged to form $R_{1}$ of size $2 N$ which includes elite members of both parent and offspring populations. All individuals in $R_{1}$ are sorted into a number of non-domination levels such as $F_{1}, F_{2}$ and so on. Starting from $F_{1}$, the next population $P_{2}$ is formed until the size of $P_{2}$ achieves $N$. The crowding distance approach is used to obtain $N$ member $P_{2}$ accepting the last level $F_{n}$ partially. This process is repeated until to reach a termination criterion (Deb et al. 2002, Sadeghi et al. 2014, Deb \& Jain, 2014.

SPEA2 is also an elitist evolutionary algorithm and works similarly. One of the main differences is that SPEA2 uses an external archive that consists of the previously found non-dominated solutions. In addition, SPEA2 uses an advanced fitness assignment strategy which considers both dominated and dominating individuals. Moreover, the nearest neighbour density measure is used in order to maintain the diversity (Zitzler et al., 2002).

IBEA, on the other hand, uses a different approach. The main idea is to compute the quality of each individual using a predetermined indicator reducing multiple objectives into a single "fitness" value. This enables the use of generic single optimisation methods and so the evolutionary algorithm however requires maintaining a set of trade-off solutions. In addition, only pairs of individuals are compared instead of considering entire pareto-front set and diversity preservation mechanism is not required (Zitzler \& Künzli, 2004).

\subsection{Performance Metrics}

The performance of Multi-objective Evolutionary Algorithms are assessed using various metrics, including the distance of the final pareto set to the global pareto-optimal front, distribution of the final pareto set with respect to the pareto-optimal front, and spread of the pareto set (Zitzler, 1999, Narukawa \& 
Rodemann 2012). When dealing with multi-objective optimisation problems, the purpose is to achieve a desirable non-dominated set. However, for a number of reasons, the assessment of results becomes difficult; i) several solutions are generated rather than one as in a single objective optimisation problem, ii) a number of runs needs to be performed to assess the performance of EAs due to their stochastic nature, iii) different entities, such as, coverage, diversity of a set of solutions could be measured and used as a guidance during the search process (Sarker \& Coello Coello, 2002). The MOEA performance metrics used in this study are explained in the following subsections.

\subsubsection{Generational Distance (GD)}

GD is a method to estimate how far the elements in solutions obtained are from $P F_{\text {global }}$ set and defined as (Veldhuizen \& Veldhuizen, 1999);

$$
\mathrm{GD}=\frac{1}{n}\left(\sum_{i=1}^{n} d_{i}^{p}\right)^{\frac{1}{p}}
$$

where $n$ is the number of solutions, $d_{i}$ is the Euclidean distance between each of solutions and the nearest member of $P F_{\text {global }}$. The value of $G D=0$ shows that all individuals generated are in $P F_{\text {global }}$ (Coello et al., 2006), hence lower the GD better the performance of an algorithm is.

\subsubsection{Inverted Generational Distance (IGD)}

The IGD metric was first proposed by Czyzak \& Jaszkiewicz (1998) calculating the distance between an objective vector and a reference point. However, the term itself, "inverted generational distance" was introduced in (Coello Coello \& Reyes Sierra, 2004, Sierra \& Coello, 2004).

$$
\mathrm{IGD}=\frac{1}{m}\left(\sum_{j=1}^{m} d_{j}^{p}\right)^{\frac{1}{p}}
$$

where $m$ is the number of vectors in $P F_{\text {global }}, d_{j}$ is the Euclidean distance between each member of $P F_{\text {global }}$ and the nearest solution obtained. The value 
of $p$ is fixed as 1 in this work. Lower the IGD better the performance of an algorithm is.

\subsubsection{Hypervolume (HV)}

Zitzler et al. (2007) proposed a hypervolume indicator (in the literature, it is found named as 'Size of the Space Covered' or 'Size of Dominated Space' by Zitzler \& Thiele (1999)). The size of a pareto-front set is computed in objective space by the non-dominated vectors and generally, the definition of hypervolume indicator is (Brockhoff et al. 2008);

$$
I_{H}(A)=\lambda\left(\bigcup_{a \in A}\left[f_{1}(a), r_{1}\right] \times \ldots \times\left[f_{m}(a), r_{m}\right]\right)
$$

where $I_{H}(A)$ denotes the hypervolume indicator of a solution set $A \subseteq X$ and it is bounded by a reference point $r=\left(r_{1}, \ldots, r_{m}\right) \in R_{m}$ while it is assumed that $m$ objective functions $f=\left(f_{1}(x), f_{2}(x), \ldots, f_{m}(x)\right)$ that map solutions $x \in X$ from the decision space $X$ to maximize the hypervolume indicator $I_{H}(A)$. The Lebesgue measure of a hypervolume set is depicted as $\lambda(H V)$ where $\left[f_{1}(a), r_{1}\right] \times$ $\left[f_{2}(a), r_{2}\right] \times \times\left[f_{m}(a), r_{m}\right]$ is the $m$-dimensional hypercuboid consisting of all points that are weakly dominated by the individual $a$ but not weakly dominated by the reference point. Unlike the other metrics used in this study, higher the $\mathrm{HV}$ better the performance of an algorithm is.

\section{Methodology}

We propose a two-stage fuzzy based optimisation approach to deal with the multi-objective integrated supply chain management problem. The first stage of suppliers are ranked using an IT2FS method while in the second stage, three MOEAs are studied to solve the supplier selection and inventory planning problem considering the information provided from the first stage.

\subsection{Stage One: Ranking of Suppliers}

In this stage, the purpose is to achieve an appropriate approach to rank vendors by identifying the criteria that plays a critical role in supplier selection, 
Table 1: Linguistic weights of the attributes represented by Interval Type-2 Fuzzy Set (Turk et al. 2014)

\begin{tabular}{ll}
\hline \hline Linguistic terms & Interval Type-2 Fuzzy Sets \\
\hline \hline Low importance & $((0.0,0.0,0.2,0.3),(0.0,0.0,0.2,0.5))$ \\
Moderate importance & $((0.3,0.4,0.4,0.5),(0.1,0.4,0.4,0.7))$ \\
High importance & $((0.5,0.6,0.6,0.7),(0.3,0.6,0.6,0.9))$ \\
Very High importance & $((0.7,0.8,1.0,1.0),(0.5,0.8,1.0,1.0))$ \\
\hline
\end{tabular}

Table 2: Linguistic performance rates represented Interval Type-2 Fuzzy Set Turk et al. 2014)

\begin{tabular}{ll}
\hline \hline Linguistic terms & Interval Type-2 fuzzy sets \\
\hline \hline Poor & $((0,0,2,3),(0,0,2,5))$ \\
Good & $((3,4,4,5),(1,4,4,7))$ \\
Very Good & $((5,6,6,7),(3,6,6,9))$ \\
Excellent & $((7,8,10,10),(5,8,10,10))$ \\
\hline
\end{tabular}

and assessing the performance of vendors for choosing suppliers Turk et al. (2015).

\subsubsection{Membership Functions}

In the study proposed by Ordoobadi (2009), decision makers have examined two attributes; importance of each criterion to evaluate vendors, performance rating of suppliers. Turk et al. (2014) developed this work, investigating uncertainty in the proposed problem using IT2FS in order to provide a guideline to choose an appropriate vendor.

Each criterion is rated using linguistic weights: 'low importance', 'moderate importance', 'high importance' and 'very high importance' (Ordoobadi, 2009). The numeric scale defined between 0 and 1 corresponds to the fuzzy numbers shown in Table 1 which indicates the IT2 membership functions used to depict each of the linguistic weights. Table 1 shows the parameters of a trapezoidal IT2FS where a trapezoidal is represented by four numbers. In this case, we do this for both the lover and upper membership functions.

Moreover, decision makers assigned each supplier performance using linguis- 
tic weights: 'excellent', 'very good', 'good' and 'poor'. The numeric scale determined between 0 and 10 corresponded to the fuzzy numbers of each criterion value (Ordoobadi, 2009, Turk et al., 2014). The IT2FS are created in the same manner as denoted previously for modelling the importance weights, and their values are demonstrated in Table 2 (Turk et al. 2014).

\subsubsection{Proposed Method for Ranking Suppliers}

After identifying the selection criteria and generating appropriate fuzzy membership functions, to measure the performance of suppliers and elicit their ranks, fuzzy mathematical operators are used to calculate a fuzzy score for each vendor and then to obtain crisp values, these scores are converted through a type-reduction and defuzzification process. Using these crisp values, the rank of supplier is achieved. For completeness, further explanation of processes are given as follows:

Firstly, each importance of criterion chosen by decision makers is used to generate trapezoidal IT2FSs. These criteria are expressed in linguistic terms with respect to experts' perceptions. For example, if a criterion's importance weight is 'high' then is assigned as $((0.5,0.6,0.6,0.7),(0.3,0.6,0.6,0.9))$ as seen in Table 1. After all criteria are converted into fuzzy numbers, all criteria on the same branch are multiplied by the previous criterion as indicated in Figure 2 Let $w_{i}$ indicates the fuzzy importance weight of criterion $i$ where $i=1,2, \ldots, 10$. For instance, $w_{5}$ is achieved by multiplying the importance weight of reliability by the importance weight of the service as:

$$
\begin{aligned}
w_{8} & =((0.5,0.6,0.6,0.7),(0.3,0.6,0.6,0.9))((0.5,0.6,0.6,0.7),(0.3,0.6,0.6,0.9)) \\
& =((0.25,0.36,0.36,0.49),(0.09,0.36,0.36,0.81))
\end{aligned}
$$

After all weights are computed in the same manner, trapezoidal IT2FSs for the performance of vendors are generated in the same way as criteria importance. And then the aggregate fuzzy set for each vendor is computed by multiplying the fuzzy performance rates matrix by the fuzzy importance weights as detailed in Turk et al. (2014). Finally, fuzzy values are converted through Centroid typereduction and defuzzification methods to crisp values in order to rank suppliers. 


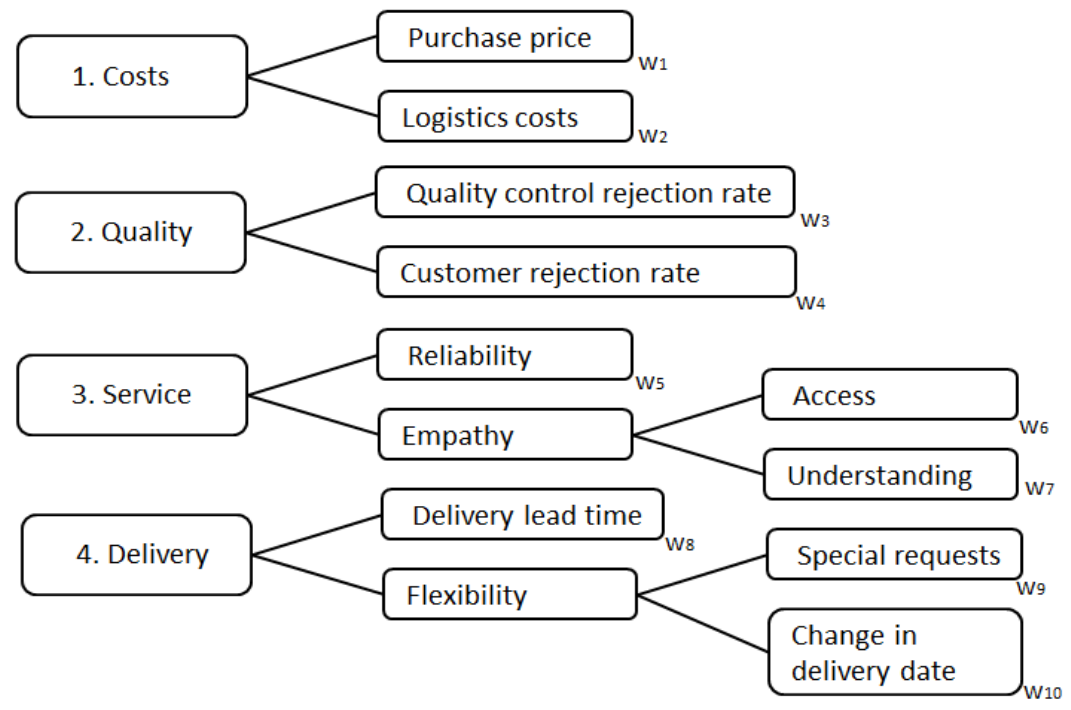

Figure 2: The criteria and sub-criteria used for selection of suppliers (Ordoobadi 2009).

\subsection{Stage Two: Inventory Planning with Consideration of Supplier Risk}

The problem addressed in this study captures features of multi-product production while considering the different components. It consists of multiple suppliers, manufacturing plants and potential customers varying from experiment to experiment. The time for planning is broken down to 'chunks' of time. The first time period depends on what the original stock levels are(Turk et al., 2015). Other assumptions:

1. Every supply can supply all plants with all components.

2. Every supply and plant has limited capacity for each component and product.

3. The cost of the whole operation is the cost of: product, order, transport, holding of inventory and stock out.

4. Distance between nodes are fixed and known.

Moreover, if an order is not in stock, stock out cost is computed and where we cannot satisfy demand, the products are bought at the full price from the 
Table 3: Notation for Decision Variables (Turk et al. 2015)

\begin{tabular}{cl}
\hline Variable & Meaning \\
\hline$P_{A}(p, j, k, t)$ & Amount of product $p$ from plant $j$ to customer $k$ in period $t$ \\
$C_{A}(c, i, j, t)$ & Amount of component $c$ from supplier $i$ to plant $j$ in period $t$ \\
$P_{I}(p, j, t)$ & Inventory of product $p$ at plant $j$ in period $t$ \\
$C_{I}(c, j, t)$ & Inventory of component $c$ at plant $j$ in period $t$ \\
\hline
\end{tabular}

competitors(Turk et al., 2015).

\subsubsection{Multi-objective Model}

Presented below is the formulation of the supply chain problem where $i, j$ and $k$ represent a supplier, manufacturing plant and customer, respectively. In addition, a product, indicated by $p$, is formed using $c$ components in discrete time period denoted by $t$. Tables 3 and 4 provide the notation to build the model.

In this work, two objectives are minimised; (i) potential risk endured $T R$ (Equation 15 as a result of the supplier selection and (ii) the total cost of the supply chain $T C$ (Equation 14).

The equation 14 computes the total cost summing up the following entities for each time period/step. In the first row of the equation, the total cost of inventory is shown for the components and products successively. The transportation cost is accumulated considering the products and then components in the second and third row, respectively. The next row adds the component order and setup costs. The manufacturing and shortage costs for each product are included in the following row. Finally, the total shortage cost for components and penalty are added to the overall. The penalty cost is incurred when the quantity of production does not satisfy the customer demands. 
Table 4: Notation for Parameters (Turk et al. 2015)

\begin{tabular}{ll}
\hline \hline Notation & Meaning \\
\hline \hline$C_{S}(c, j)$ & Initial stock for component $c$ at plant $j$ \\
$P_{S}(p, j)$ & Initial stock for product $p$ at plant $j$ \\
$X_{C}(c, i)$ & Component $c$ 's capacity of supplier $i$ \\
$X_{P}(p, j)$ & Product $p$ 's capacity at plant $j$ \\
$Y_{C}(c, i)$ & Component $c$ 's cost of supplier $i$ \\
$Y_{P}(p, k)$ & Product $p$ 's selling price for customer $k$ \\
$T_{C}(c, i, j)$ & Carrying cost for component $c$ between supplier $i$ and plant $j$ \\
$T_{P}(p, j, k)$ & Carrying cost for product $p$ between plant $j$ and customer $k$ \\
$I_{C}(c, j)$ & Component $c$ 's inventory cost at plant $j$ \\
$I_{P}(p, j)$ & Product $p$ 's inventory cost at plant $j$ \\
$S_{C}(c, j)$ & Shortage cost at plant $j$ for component $c$ \\
$S_{P}(p, j)$ & Shortage cost at plant $j$ for product $p$ \\
$O_{C}(c, i)$ & Ordering cost of supplier $i$ for component $c$ \\
$M_{P}(p, j)$ & Manufacturing cost for product $p$ at plant $j$ \\
$S(p, j)$ & Setup cost in plant $j$ for product $p$ \\
$H(p, j)$ & Holding cost percentage for product $p$ at plant $j$ \\
$D_{S}(i, j)$ & Distance between supplier $i$ and plant $j$ \\
$D_{P}(j, k)$ & Distance between plant $j$ and customer $k$ \\
$R a n k(i)$ & Rank of vendor $i$ \\
$R i s k(i)$ & Risk of vendor $i$ \\
$D(p, k, t)$ & Customer $k$ demand for product $p$ in each period $t$ \\
$P_{M}(p, k, t)$ & Non-fulfilment amount of product $p$ for customer $k$ in period $t$ \\
\hline &
\end{tabular}

$$
\begin{aligned}
T C= & \sum_{t}\left(\sum_{p} \sum_{j} I_{P}(p, j) \times P_{I}(p, j, t)+\sum_{c} \sum_{j} I_{C}(c, j) \times C_{I}(c, k, t)\right. \\
& +\sum_{p} \sum_{j} \sum_{k}\left(P_{A}(p, j, k, t) \times D_{P}(j, k) \times T_{P}(p, j, k)\right) \\
& +\sum_{c} \sum_{i} \sum_{j}\left(C_{A}(c, i, j, t) \times D_{S}(i, j) \times T_{C}(c, i, j)\right) \\
& +\sum_{c} \sum_{i} \sum_{j} O_{C}(c, i) \times C_{A}(c, i, j, t)+\sum_{p} \sum_{j} \sum_{k} S(p, j) \times P_{A}(p, j, k, t) \\
& +\sum_{p} \sum_{j} \sum_{k} M_{P}(p, j) \times P_{A}(p, j, k, t)+\sum_{p} \sum_{j} S_{P}(p, j) \times P_{I}(p, j, t) \\
& \left.+\sum_{c} \sum_{j} S_{C}(c, j) \times C_{I}(c, j, t)+\sum_{p} \sum_{k} P_{M}(p, k, t) Y_{P}(p, k)\right) .
\end{aligned}
$$




$$
T R=\sum_{t} \sum_{c} \sum_{i} \sum_{j} C_{A}(c, i, j, t) \times R i s k(i)
$$

Equation 15 demonstrates total risk of suppliers with respect to Equation 16 which shows the calculation of a coefficient for the risk of each supplier by normalising the supplier rank indicated in Table 7.

$$
\operatorname{Risk}(i)=\frac{\sum_{i} \operatorname{Rank}(i)}{\operatorname{Rank}(i)}
$$

Equation 17 depicts the supplier capacity for each period and Equation 18 shows the capacity of the plant for each period.

$$
\begin{array}{ll}
\sum_{j} C_{A}(c, i, j, t) \leq X_{C}(c, i) & \text { for } \forall c, i, t \\
\sum_{k} P_{A}(p, j, k, t) \leq X_{P}(p, j) & \text { for } \forall p, j, t
\end{array}
$$

Equation 19 shows that demand is satisfied by the production units and if, the production units are not less than the order amount of customer, it is provided from retailers explained as non-fulfilment amount of a product in Table 4 . And Equation 20 guarantees that the production units are not more than the order amount of customer. In this model, it is assumed that the first product composes of the first and second components and the second one is produced using the third and fourth components. Equation 21 describes the inventory-control constraints for these components and Equation 22 represents inventory-control constraints for each product.

$$
\begin{gathered}
P_{M}(p, k, t)=D(p, k, t)-\sum_{j} P_{A}(p, j, k, t) \quad \text { for } \forall p, k, t . \\
\sum_{j} P_{A}(p, j, k, t) \leq D(p, k, t) \quad \text { for } \forall p, k, t .
\end{gathered}
$$




$$
\sum_{i} C_{A}(c, i, j, t)+C_{S}(c, j)=\sum_{k} P_{A}(p, j, k, t)+P_{I}(p, j, t)+C_{I}(c, j, t)
$$

for $\forall j, t$ where $p=1$ for $c=\{1,2\}$ and where $p=2$ for $c=\{3,4\}$.

$$
\begin{aligned}
\sum_{j} \sum_{k} P_{A}(p, j, k, t)+\sum_{j} P_{S}(p, j) & =\sum_{k} D(p, k, t)+\sum_{j} P_{I}(p, j, t) & \\
& +\sum_{k} P_{M}(p, k, t) & \text { for } \forall p, t .
\end{aligned}
$$

Turk et al. (2015) studied two generic single point based heuristic optimisation algorithms, each using a different scalarisation method to solve the two-objective problem. That study illustrated the multi-objective nature of the problem testing the proposed approaches on a simple single problem instance. This study extends the previous work and investigates three proper multi-objective meta-heuristic algorithms to solve the problem with an attempt to detect the best performing approach.

\section{Preliminary Experiments}

In this section, we cover the common experimental and algorithmic design, problem instances and their characteristics as well as preliminary experiments discussing the results from application of stage one approach and parameter tuning of NSGA-II, SPEA2 and IBEA.

\subsection{Experimental Design}

We implemented a fuzzy model and run the stage one approach resulting with risk of using a particular supplier as explained in Section 3.1. Then this information is fed into the multi-objective evolutionary algorithms to solve the integrated problem of supplier selection and inventory planning.

The Jmetal suite (Durillo \& Nebro, 2011; Durillo et al. 2010) is used to run all experiments with the multi-objective evolutionary algorithms. Each trial is repeated for 30 times during the experiments, where each run yields a set of 
trade-off solutions. A run terminates whenever the 5000 iterations/generations are exceeded.

A real-valued chromosome representation is used to represent a potential inventory plan. This plan is encoded into a 4 dimensional array. Each dimension points out the source node, destination node, component/product and time period, successively. Each array entry contains a value $\in[0,1]$, representing the ratio of raw material or goods added to the inventory with respect to the full capacity of the chosen product at a given source and destination node within a specific time period. For instance, if the value of currentPlan $[3,1,4,2]$ is 0.5 , this would demonstrate that in period 2 , source node 3 is holding $50 \%$ of its capacity of product 4 for destination node 1 . The holding can never exceed $100 \%$ within any time period with the proposed encoding. In addition to this, order amount are decided in certain increments starting from a specified minimum value. For example, assuming an increment of 100 units and a minimum order of 300 units for a particular product at a particular node, the orders are restricted to the increments of 100 starting from 300 (e.g. 300, 400, 500 etc.).

The initial population is generated randomly. A binary tournament selection is employed to create a offspring population. Simulated Binary (SBX) Crossover and Polynomial Mutation operator are used by all MOEAs. The common parameters of SPEA2 and IBEA include population size $(P)$, crossover probability $\left(P_{c}\right)$, distribution index for crossover $\left(D_{m}\right)$, distribution index for mutation $\left(D_{c}\right)$ and archive size $(A)$. NSGA-II has the same algorithmic control parameters, excluding the archive size. Crossover and mutation probability is utilised to maintain the frequency of operations. Distribution index for crossover and mutation are used to control the spread of offspring solutions for which larger values support "nearer parent" solutions. All the algorithmic control parameters are tuned for each particular algorithm.

\subsection{Problem Instances}

In this study, four groups of six problem instances are used, totalling up to twenty four instances (Table 6). For each instance, five suppliers denoted 

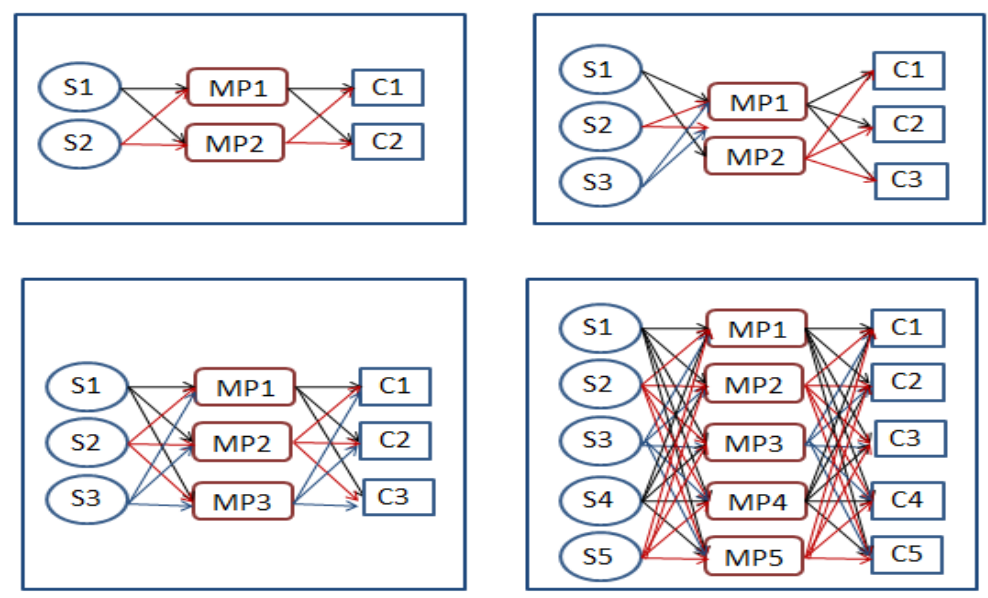

Figure 3: Representation of systems consisting of fixed number of suppliers, manufacturing plants and customers, namely $2 \times 2 \times 2,3 \times 2 \times 3,3 \times 3 \times 3$ and $5 \times 5 \times 5$ that 24 instances are derived from.

from S1 to S5 are considered for simplicity. Each group is formed of fixed number of suppliers, manufacturing plants and customers, namely $2 \times 2 \times 2$, $3 \times 2 \times 3,3 \times 3 \times 3$ and $5 \times 5 \times 5$ as illustrated in Figure 3 . The flow of goods is also shown in Figure 3 using arrows. The planning horizon contains three discrete time periods. The production cost, capacity, minimum order quantity, order quantity and initial stock (see Section 3 for more details) are all parametrised for the instances. Two different settings are used for the problem instances as summarised in Table 5. The first instances of each group, namely Inst1, Inst7, Inst13 and Inst19 are real-world problem instances (Miller et al. 2012) and use a fixed parameter setting. The remaining instances are randomly generated using a certain range for each parameter setting as shown in Table 6 The problem instances will be made publically available from https : //www.researchgate.net/publication/311873391_SCMproblem. 
Table 5: Configuration of problem instance parameters

\begin{tabular}{|l|l|l|l|l|l|l|l|l|l|l|l|l|}
\hline \multirow{2}{*}{$\begin{array}{l}\text { Node } \\
\text { Configuration }\end{array}$} & \multirow{2}{*}{ Label } & \multicolumn{3}{|c|}{$\begin{array}{c}\text { Production Cost } \\
\end{array}$} & & \multicolumn{2}{|c|}{ Capacity } & \multicolumn{2}{c|}{$\begin{array}{c}\text { Minimum Order } \\
\text { Quantities }\end{array}$} & \multicolumn{2}{c|}{ Order Quantities } & \multicolumn{2}{c|}{ Initial Stock } \\
\cline { 3 - 14 } & & Components & Products & Components & Products & Components & Products & Components & Products & Components & Products \\
\hline Fixed & $\mathrm{C} 1$ & $(0.9,0.15,0.3,0.5)$ & $(0.5,0.2)$ & 1000 & 1000 & 100 & 100 & 100 & 100 & 250 & 250 \\
\hline Random & $\mathrm{C} 2$ & {$[0.20,0.80]$} & {$[0.20,0.80]$} & {$[500,1000]$} & {$[500,1000]$} & {$[50,200]$} & {$[50,200]$} & {$[10,50]$} & {$[10,50]$} & {$[0,500]$} & {$[0,500]$} \\
\hline
\end{tabular}

Table 6: Characteristics of the problem instances (S: the number of suppliers, P: the number of manufacturing plants, C: the number of customers, CO: configuration)

\begin{tabular}{|c|c|c|c|c|c|c|c|c|c|c|c|c|c|c|c|c|c|c|c|}
\hline Inst & S & $\mathrm{P}$ & $\mathrm{C}$ & $\mathrm{CO}$ & Inst & $\mathrm{S}$ & $\mathrm{P}$ & $\mathrm{C}$ & $\mathrm{CO}$ & Inst & S & $\mathrm{P}$ & $\mathrm{C}$ & $\mathrm{CO}$ & Inst & S & $\mathrm{P}$ & $\mathrm{C}$ & $\mathrm{CO}$ \\
\hline Inst1 & 2 & 2 & 2 & $\mathrm{C} 1$ & Inst7 & 3 & 2 & 3 & $\mathrm{C} 1$ & Inst13 & 3 & 3 & 3 & $\mathrm{C} 1$ & Inst19 & 5 & 5 & 5 & $\mathrm{C} 1$ \\
\hline Inst2-7 & 2 & 2 & 2 & $\mathrm{C} 2$ & Inst8-13 & 3 & 2 & 3 & $\mathrm{C} 2$ & Inst14-19 & 3 & 3 & 3 & $\mathrm{C} 2$ & Inst20-24 & 5 & 5 & 5 & $\mathrm{C} 2$ \\
\hline
\end{tabular}

\subsection{Results from Ranking of Suppliers}

Although the overall two-stage approach operates in an integrated manner, we report the results from stage one separately for the ease of empirical analysis. The stage one fuzzy method for ranking of relevant suppliers for each instance is executed as explained in Section 3.1, yielding an output of score, ranking and risk for each supplier as shown in Table 7. Depending on the number of suppliers in the associated problem instance, the results obtained from the IT2FS approach for the relevant suppliers are used in the next stage. For example, considering Inst1, where $S=2$, associated risk values for S1 and S2 are fed into the stage two approach.

\subsection{Parameter Tuning of NSGA-II, SPEA2 and IBEA}

The Taguchi orthogonal arrays (Taguchi \& Yokoyama, 1993) as a design of experiments method is used for parameter tuning of each MOEA for improved performance. We investigated four control parameters for NSGA-II with the following potential settings: $P \in\{25,50,100,200\}, P_{c} \in\{0.6,0.7,0.8,0.9\}, D_{c}$ and $D_{m} \in\{5,10,15,20\}$, and five control parameters for SPEA2 and IBEA with the addition of $A \in\{25,50,100,200\}$. The best parameter configuration is determined based on the $L^{16}$ Taguchi orthogonal arrays design.

We have arbitrarily chosen three instances of different sizes: $\{$ Inst1, Inst13, 
Table 7: Rank and Risk Values of Suppliers for each Instance

\begin{tabular}{|c|c|c|c|c|c|c|c|}
\hline \hline \multirow{2}{*}{ Suppliers } & \multirow{2}{*}{ Crisp Score } & \multicolumn{2}{|c|}{ Inst1-Inst6 } & \multicolumn{2}{|c|}{ Inst7-Inst18 } & \multicolumn{2}{|c|}{ Inst19-Inst24 } \\
\cline { 3 - 9 } & & Rank & Risk & Rank & Risk & Rank & Risk \\
\hline S1 & 10.88 & 2 & 3.28 & 3 & 4.90 & 4 & 6.60 \\
S2 & 24.80 & 1 & 1.44 & 1 & 2.15 & 1 & 2.90 \\
S3 & 17.59 & - & - & 2 & 3.03 & 2 & 4.10 \\
S4 & 5.96 & - & - & - & - & 5 & 12.05 \\
S5 & 12.56 & - & - & - & - & 3 & 5.72 \\
\hline
\end{tabular}

Inst19\} and Each of the sixteen parameter settings as provided in Table 8 is tested using each MOEA applying to the selected problem instances as required by the $L^{16}$ Taguchi orthogonal array design. In order to assess the performance of each setting for an MOEA, mean rank (per run), which is obtained by ranking each setting with respect to hypervolume of the pareto set for each run and then averaging the ranks of a setting over all runs and problem instances. A lower value indicates a better performance. As an example in Table 8 the parameter settings and average rank of three MOEAs are shown.

A mean rank for a particular parameter value setting to indicate its main effect is computed by taking the average of the rank of all runs with that setting on all instances. For example, let us consider the mean effect of a population size 25 for NSGA-II, which gets computed as $(14.87+12.14+12.27+11.00) / 4=12.60$ where this parameter setting corresponds to the first 4 in Table 8 . Figure 4 provides the main effects plot indicating the performance of each parameter value setting. The best configuration for NSGA-II is attained as 200 for $P$, 0.9 for $P_{c}, 20$ for $D_{c}$ and 10 for $D_{m}$. Moreover, ANOVA is utilised to analyse the contribution of each parameter setting on the performance of MOEAs. Table 9 summarises the results. The population size as well as distribution index for crossover, distribution index for mutation, and population size parameter 
Table 8: Average rank for three multi-objective algorithms, with a particular parameter configuration based on the $L^{16}$ Taguchi orthogonal array

\begin{tabular}{lcccccccc}
\hline \hline \multirow{2}{*}{$\begin{array}{l}\text { nxperiment } \\
\text { number }\end{array}$} & $\mathrm{P}$ & $P_{c}$ & $D_{c}$ & $D_{m}$ & $A$ & \multicolumn{3}{c}{ Average Rank } \\
\hline 1 & & & & & & NSGA-II & SPEA2 & IBEA \\
2 & 0.6 & 5 & 5 & 25 & 14.87 & 15.8 & 15.0 \\
3 & 25 & 0.7 & 10 & 10 & 50 & 12.14 & 11.3 & 2.6 \\
4 & 25 & 0.8 & 15 & 15 & 100 & 12.27 & 6.0 & 10.5 \\
5 & 25 & 0.9 & 20 & 20 & 200 & 11.00 & 4.8 & 5.0 \\
6 & 50 & 0.6 & 10 & 15 & 200 & 10.80 & 13.6 & 6.4 \\
7 & 50 & 0.7 & 5 & 20 & 100 & 11.84 & 10.2 & 8.5 \\
8 & 50 & 0.8 & 20 & 5 & 50 & 8.59 & 6.3 & 5.0 \\
9 & 50 & 0.9 & 15 & 10 & 25 & 7.86 & 5.8 & 11.6 \\
10 & 100 & 0.6 & 15 & 20 & 50 & 6.71 & 11.1 & 7.6 \\
11 & 100 & 0.7 & 20 & 15 & 25 & 4.74 & 11.4 & 13.7 \\
12 & 100 & 0.8 & 5 & 10 & 200 & 9.52 & 6.5 & 4.8 \\
13 & 100 & 0.9 & 10 & 5 & 100 & 6.09 & 2.8 & 11.8 \\
14 & 200 & 0.6 & 20 & 10 & 100 & 3.79 & 10.1 & 9.2 \\
15 & 200 & 0.7 & 15 & 5 & 200 & 4.43 & 9.9 & 8.0 \\
16 & 200 & 0.8 & 10 & 20 & 25 & 4.53 & 7.1 & 12.8 \\
\hline \multirow{2}{*}{15} & 200 & 0.9 & 5 & 15 & 50 & 6.80 & 3.3 & 3.4 \\
\hline
\end{tabular}

Table 9: ANOVA test results for dismissing the contribution of each parameter for MOEAs in terms of percent contribution

\begin{tabular}{l|cccccc|c}
\hline \hline MOEAs & $P$ & $P_{c}$ & $D_{c}$ & $D_{m}$ & $A$ & Error & Total \\
\hline NSGA-II & 79.28 & 1.64 & 17.93 & 0.12 & - & 1.03 & $100 \%$ \\
SPEA2 & 8.07 & 4.46 & 0.17 & 86.46 & 0.84 & 0 & $100 \%$ \\
IBEA & 84.41 & 2.65 & 7.82 & 2.85 & 2.27 & 0 & $100 \%$ \\
\hline
\end{tabular}

settings have significant contribution within a confidence level of $95 \%$ on the performance of NSGA-II, SPEA2 and IBEA, respectively.

In the same manner, the parameters of the other two MOEAs are tuned and the results achieved are depicted in Table 10. For three instances, the best parameters setting is performed to confirm that optimum parameters setting is found using the Taguchi method. Based on the hypervolume values, the tuned 

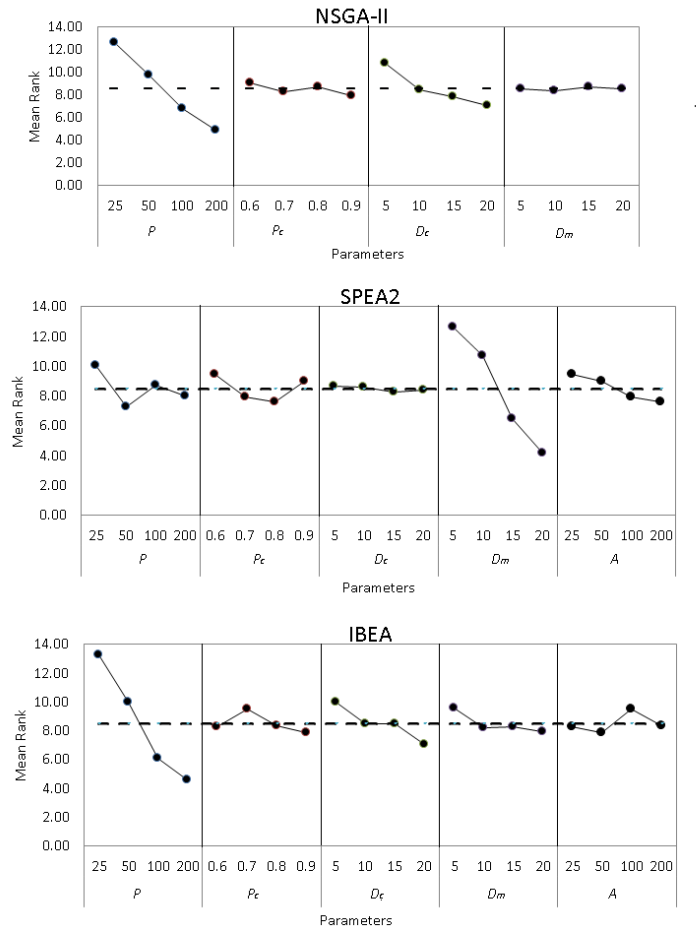

Figure 4: Main effects plot with mean rank values in three multi-objective algorithms

Table 10: Tuned Parameters of three MOEAs

\begin{tabular}{lccccc}
\hline \hline MOEAs & $\mathrm{P}$ & $P_{c}$ & $D_{c}$ & $D_{m}$ & $\mathrm{~A}$ \\
\hline NSGA-II & 200 & 0.9 & 20 & 10 & - \\
SPEA2 & 50 & 0.8 & 15 & 20 & 200 \\
IBEA & 200 & 0.9 & 20 & 20 & 50 \\
\hline
\end{tabular}

three MOEAs outperform all the other setting with the highest hypervolume values for each instance.

\section{Computational Results for Inventory Planning with Consideration}

\section{of Supplier Risk}

NSGA-II, SPEA2 and IBEA are applied to the twenty four problem instances as a part of the stage two to discover high quality inventory plans based on 
the described multi-objective model (See Section 3.2). These MOEAs provide flexibility for the decision makers enabling them to choose a solution from a set of 'equal' quality solutions reflecting the different levels of trade-off between the total supplier risk and cost of the supply chain operation.

Table 11 provides the performance comparison of all MOEAs across all problem instances based on hypervolume, generational distance and inverse generational distance. We have further performed statistical analysis of results based on Wilcoxon signed-rank test. In the overall, NSGA-II is the best performing multi-objective algorithm on average across all instances in terms of all metrics as shown in Table 11 and we use the following notation in the table.

$$
\begin{array}{ll}
H_{0}: & P_{N S G A-I I}>P_{O} \\
H_{A}: & P_{N S G A-I I} \neq P_{O}
\end{array}
$$

where $H_{0}$ represents null hypothesis which asserts that the probability distributions of the pareto-optimal solutions for NSGA-II are better than other multi-objective algorithm $O$ and $H_{A}$ represents alternative hypothesis which the distributions of results differ for NSGA-II and the multi-objective algorithm considered. The confidence level (significance level) for the non-parametric test of Wilcoxon signed-rank is set to $95 \%$ (p-value under 0.05). The following notations are used in Table 11. Let us consider two algorithms; NSGA-II versus S, $>(<)$ indicates that NSGA-II $(\mathrm{S})$ is better than S (NSGA-II) and this performance difference is statistically significant within a confidence interval of $95 \%$ and NSGA-II $\geq S($ NSGA-II) $\leq S$ ) denotes that NSGA-II (S) performs slightly better on average than S (NSGA-II) with no statistical significance.

NSGA-II performs significantly better than SPEA2 and IBEA based on hypervolume on all and fourteen out of twenty four instances, respectively. As for the remaining instances, NSGA-II is slightly better than IBEA on Inst1, Inst14, Inst16 and IBEA is slightly better than NSGA-II for seven instances. When the size of problem is enlarged, NSGA-II still provides significantly better hypervolume values than the other two algorithms achieved (shown as Figure 5 
Secondly, with respect to the generational distance, NSGA-II performs significantly better than SPEA2 and IBEA for twenty three and fourteen out of twenty four instances, respectively. For Inst4, SPEA2 is slightly better than NSGA-II. IBEA is slightly better than NSGA-II over ten instances achieving lower values of generational distance. NSGA-II provides significantly better results as the size of the problem grows.

Finally, considering inverse generational distance, NSGA-II outperforms SPEA2 and IBEA for all and seventeen out of twenty four instances, respectively. This performance variation is statistically significant. IBEA is slightly better than NSGA-II on seven instances. NSGA-II produces better results when compared to SPEA2 and IBEA across the last relatively large six instances formed by the $5 \times 5 \times 5$ model.

As a sample, we plotted the pareto-front achieved by each MOEA on one small and one relatively large arbitrarily chosen instances of Inst1 (Figure 5(a) and Inst19 (Figure 5(b)) . As it can be observed from Figure 5(a). NSGA-II and IBEA produce a wider spread of solutions on the pareto- front when compared to SPEA2. However, when the size of problem gets larger, the spread of paretofront achieved by NSGA-II is better than the others as illustrated in Figure 5(b)

The proposed multi-objective approach provides means to the decision makers to select a solution among multiple trade-off solutions. A common way of (automatically) reducing all solutions into a 'preferable' reasonable single solution is detecting the solution at the knee point on the pareto-front. We have used the method presented in (Bechikh et al. 2010) to obtain the a single solution based on the knee point for the selected problem instances of Inst2, Inst7, Inst13 and Inst23 from each group of instances. The total cost $(T C)$ and risk $(T R)$ objectives computed for each such solution to each instance is summarised in Table 12 which also provides all constituent costs considered.

We have observed that all three MOEAs achieved knee solutions for the majority of the instances satisfying at least $95 \%$ of the customer demand. In our model, we have merged all cost related entities into a single quantity. The results indicate that there could be trade-off in between different constituent 
(a)

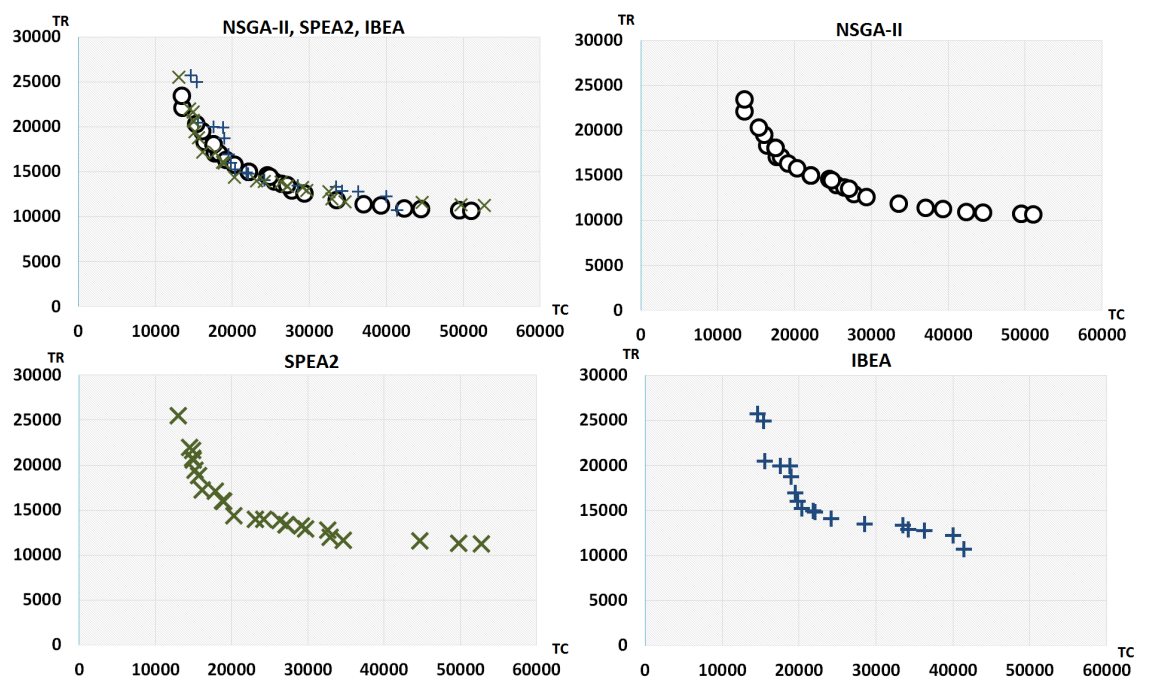

(b)

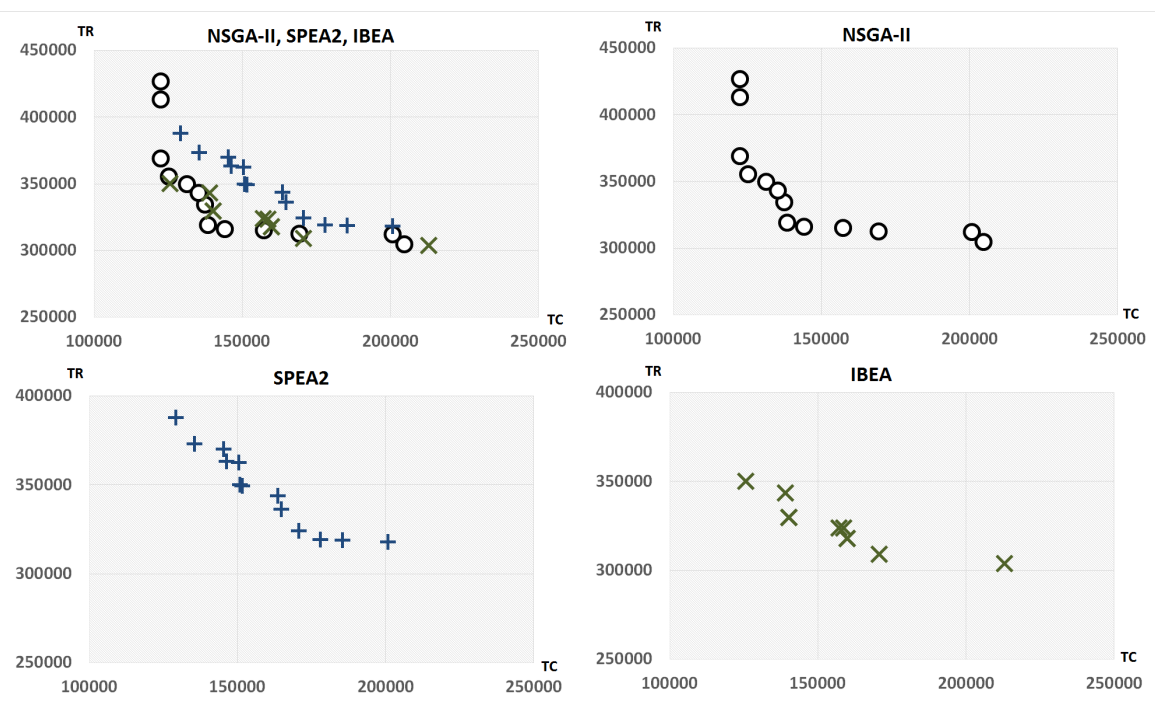

Figure 5: Pareto-front plots for NSGA-II, SPEA2 and IBEA based on $T R$ vs $T C$ from a sample run on (a) Inst1 and (b) Inst19. 
Table 11: Performance comparison of NSGA-II, SPEA2 and IBEA for the two-objective supply chain problem based on three metrics

\begin{tabular}{|c|c|c|c|c|c|c|c|c|c|c|c|c|c|c|c|c|}
\hline & \multicolumn{5}{|c|}{ HyperVolume } & \multicolumn{5}{|c|}{$\begin{array}{c}\text { Generational } \\
\text { Distance }\end{array}$} & \multicolumn{5}{|c|}{$\begin{array}{l}\text { Inverse Generational } \\
\text { Distance }\end{array}$} \\
\hline & & \multirow{2}{*}{$\begin{array}{r}\text { NSGA-II } \\
0.7670\end{array}$} & \multirow{3}{*}{$>$} & \multirow{3}{*}{\begin{tabular}{r|} 
SPEA2 \\
0.7499 \\
0.006
\end{tabular}} & \multirow{3}{*}{$\geq$} & \multirow{3}{*}{$\begin{array}{r}\text { IBEA } \\
0.7640 \\
0.007 \\
\end{array}$} & \multirow{3}{*}{$\begin{array}{r}\text { NSGA-II } \\
38,788.8 \\
14,576.7 \\
\end{array}$} & \multirow{3}{*}{$>$} & \multirow{3}{*}{\begin{tabular}{r|} 
SPEA2 \\
$46,646.3$ \\
$12,136.2$ \\
\end{tabular}} & \multirow{3}{*}{$>$} & \multirow{3}{*}{\begin{tabular}{|r|} 
IBEA \\
$38,853.6$ \\
$15,011.4$ \\
\end{tabular}} & \multirow{3}{*}{\begin{tabular}{r|} 
NSGA-II \\
$80,868.5$ \\
$8,937.1$ \\
\end{tabular}} & \multicolumn{2}{|r|}{ SPEA2 } & & IBEA \\
\hline & Mean & & & & & & & & & & & & $>$ & $112,660.2$ & $>$ & $81,344.6$ \\
\hline mout & Stnd. & 0.007 & & & & & & & & & & & & $9,624.3$ & & $7,693.6$ \\
\hline & Mean & 0.7524 & $>$ & 0.7443 & $>$ & 0.7522 & $21,050.6$ & $>$ & $20,784.4$ & $\leq$ & $17,795.4$ & $28,626.2$ & $>$ & $38,201.2$ & $\leq$ & $28,466.5$ \\
\hline mole & Stnd. & 0.006 & & 0.006 & & 0.005 & $7,700.8$ & & $10,081.2$ & & $8,934.7$ & $3,469.5$ & & $8,462.3$ & & $6,151.6$ \\
\hline & Mean & 0.6798 & $>$ & 0.6690 & $\leq$ & 0.6827 & $16,155.0$ & $>$ & $23,343.1$ & $>$ & $17,111.6$ & $43,813.0$ & $>$ & $58,283.2$ & $\leq$ & $42,289.5$ \\
\hline insts & Stnd. & 0.010 & & 0.009 & & 0.007 & $7,530.8$ & & $13,191.1$ & & $8,875.5$ & $13,496.2$ & & $11,558.3$ & & $11,106.2$ \\
\hline & Mean & 0.7118 & $>$ & 0.6975 & $>$ & 0.7102 & $25,067.7$ & $\leq$ & $24,448.8$ & $\leq$ & $19,732.0$ & $19,232.5$ & $>$ & $27,137.7$ & $\leq$ & $17,924.7$ \\
\hline mitst & Stnd. & 0.007 & & 0.007 & & 0.007 & $11,364.0$ & & $11,204.9$ & & $6,863.9$ & $4,192.6$ & & $5,090.4$ & & $3,050.3$ \\
\hline Jat & Mean & 0.7389 & $>$ & 0.7261 & $\leq$ & 0.7419 & $29,973.3$ & $>$ & $35,671.8$ & $\leq$ & $27,021.3$ & $32,679.3$ & $>$ & $44,841.3$ & $\leq$ & $29,225.3$ \\
\hline Sto & Stnd. & 0.006 & & 0.006 & & 0.007 & $15,064.0$ & & $13,572.4$ & & $11,915.6$ & $7,658.2$ & & $5,438.9$ & & $6,255.5$ \\
\hline Jnst 6 & Mean & 0.7231 & $>$ & 0.7139 & $\leq$ & 0.7262 & $19,643.4$ & $>$ & $27,964.4$ & $>$ & $20,936.1$ & $55,117.9$ & $>$ & $65,028.8$ & $\leq$ & $48,949.9$ \\
\hline & Stnd. & 0.007 & & 0.008 & & 0.008 & $8,812.7$ & & $11,106.9$ & & $9,802.2$ & $12,730.1$ & & $15,851.2$ & & $10,339.5$ \\
\hline 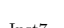 & Mean & 0.7950 & $>$ & 0.7807 & $>$ & 0.7937 & $73,194.2$ & $>$ & $98,454.5$ & $\leq$ & $68,210.8$ & $46,447.7$ & $>$ & $71,969.3$ & $>$ & $48,982.1$ \\
\hline & Stnd. & 0.006 & & 0.007 & & 0.005 & $22,948.1$ & & $27,730.4$ & & $28,363.9$ & $9,400.8$ & & $9,990.9$ & & $8,734.1$ \\
\hline Jote & Mean & 0.7963 & $>$ & 0.7890 & $\leq$ & 0.7971 & $51,292.6$ & $>$ & $65,146.7$ & $\leq$ & $44,247.6$ & $44,620.1$ & $>$ & $65,960.0$ & $>$ & $46,190.9$ \\
\hline & Stnd. & 0.003 & & 0.003 & & 0.003 & $20,828.8$ & & $18,818.9$ & & $15,131.2$ & $5,758.3$ & & $7,674.1$ & & $6,641.7$ \\
\hline & Mean & 0.8167 & $>$ & 0.8099 & $\leq$ & 0.8179 & $41,184.7$ & $>$ & $51,616.5$ & $\leq$ & $40,559.6$ & $43,557.1$ & $>$ & $63,681.4$ & $>$ & $44,826.9$ \\
\hline mstrg & Stnd. & 0.005 & & 0.005 & & 0.004 & $12,590.0$ & & $14,988.6$ & & $14,571.4$ & $7,275.9$ & & $5,910.5$ & & $5,746.3$ \\
\hline & Mean & 0.7895 & $>$ & 0.7820 & $\leq$ & 0.7902 & $50,470.7$ & $>$ & $55,166.1$ & $\leq$ & $38,779.9$ & $58,429.9$ & $>$ & $81,430.4$ & $\leq$ & $56,579.2$ \\
\hline misto & Stnd. & 0.003 & & 0.003 & & 0.004 & $19,588.8$ & & $24,624.4$ & & $10,963.8$ & $9,340.2$ & & $9,852.3$ & & $6,743.8$ \\
\hline & Mean & 0.7869 & $>$ & 0.7803 & $>$ & 0.7867 & $37,727.4$ & $>$ & $69,514.3$ & $>$ & $52,187.0$ & $37,494.6$ & $>$ & $58,278.5$ & $>$ & $41,695.2$ \\
\hline mate & Stnd. & 0.006 & & 0.006 & & 0.004 & $15,863.5$ & & $23,270.4$ & & $20,171.1$ & $6,786.3$ & & $6,945.6$ & & $3,979.2$ \\
\hline & Mean & 0.8042 & $>$ & 0.7964 & $>$ & 0.8020 & $34,812.8$ & $>$ & $42,102.8$ & $\leq$ & $31,673.3$ & $54,967.0$ & $>$ & $79,563.3$ & $>$ & $57,055.7$ \\
\hline mitud & Stnd. & 0.004 & & 0.004 & & 0.004 & $11,956.9$ & & $16,960.9$ & & $15,861.2$ & $8,548.7$ & & $9,003.0$ & & $11,706.3$ \\
\hline & Mean & 0.6995 & $>$ & 0.6844 & $>$ & 0.6970 & $65,594.3$ & $>$ & $95,628.9$ & $\leq$ & $62,425.4$ & $101,000.3$ & $>$ & $160,100.0$ & $>$ & $114,165.6$ \\
\hline & Stnd. & 0.006 & & 0.008 & & 0.010 & $32,003.3$ & & $49,046.4$ & & $28,715.9$ & $18,361.7$ & & $24,095.1$ & & $23,797.7$ \\
\hline & Mean & 0.6984 & $>$ & 0.6888 & $\geq$ & 0.6983 & $49,654.3$ & $>$ & $83,012.7$ & $>$ & $54,136.2$ & $49,903.8$ & $>$ & $84,339.3$ & $>$ & $55,597.6$ \\
\hline stit & Stnd. & 0.004 & & 0.005 & & 0.006 & $28,079.0$ & & $26,854.8$ & & $25,396.3$ & $11,236.8$ & & $14,165.9$ & & $9,390.5$ \\
\hline & Mean & 0.7193 & $>$ & 0.7072 & $>$ & 0.7164 & $51,341.3$ & $>$ & $71,084.4$ & $>$ & $53,453.3$ & $56,462.2$ & $>$ & $83,328.4$ & $>$ & $58,799.3$ \\
\hline & Stnd. & 0.003 & & 0.006 & & 0.006 & $24,657.5$ & & $35,538.5$ & & $25,487.2$ & $10,613.4$ & & $10,306.7$ & & $9,442.5$ \\
\hline & Mean & 0.6989 & $>$ & 0.6866 & $\geq$ & 0.6988 & $42,731.6$ & $>$ & $68,389.7$ & $\leq$ & $41,797.0$ & $65,099.6$ & $>$ & $90,003.3$ & $\leq$ & $64,687.5$ \\
\hline & Stnd. & 0.007 & & 0.005 & & 0.007 & $22,905.0$ & & $31,368.8$ & & $20,417.5$ & $14,635.1$ & & $13,199.8$ & & $13,303.7$ \\
\hline & Mean & 0.6937 & $>$ & 0.6818 & $\leq$ & 0.6940 & $36,036.0$ & $>$ & $49,477.5$ & $>$ & $43,127.5$ & $47,349.5$ & $>$ & $68,096.5$ & $>$ & $49,781.2$ \\
\hline & Stnd. & 0.006 & & 0.005 & & 0.004 & $18,783.2$ & & $25,172.4$ & & $21,004.7$ & $9,664.4$ & & $8,401.4$ & & $8,492.4$ \\
\hline$J_{1}$ & Mean & 0.7274 & $>$ & 0.7160 & $>$ & 0.7248 & $55,019.1$ & $>$ & $86,545.5$ & $>$ & $59,281.9$ & $38,229.7$ & $>$ & $60,904.3$ & $>$ & $42,108.6$ \\
\hline mistio & Stnd. & 0.006 & & 0.006 & & 0.005 & $25,699.5$ & & $25,917.0$ & & $21,631.8$ & $8,980.9$ & & $6,022.9$ & & $6,144.5$ \\
\hline Jat1 & Mean & 0.7007 & $>$ & 0.6896 & $>$ & 0.6994 & $207,552.1$ & $>$ & $312,479.3$ & $>$ & $255,933.3$ & $202,000.0$ & $>$ & $340,033.3$ & $>$ & $239,933.3$ \\
\hline motis & Stnd. & 0.005 & & 0.005 & & 0.005 & $85,716.5$ & & $118,172.5$ & & $92,387.1$ & $36,561.7$ & & $42,619.0$ & & $31,514.6$ \\
\hline & Mean & 0.7307 & $>$ & 0.7208 & $>$ & 0.7271 & $220,267.2$ & $>$ & $350,882.1$ & $>$ & $305,253.8$ & $95,429.6$ & $>$ & $133,300.0$ & $>$ & $107,733.7$ \\
\hline mintzo & Stnd. & 0.004 & & 0.005 & & 0.005 & $93,103.3$ & & $155,641.5$ & & $133,632.4$ & $22,586.7$ & & $22,557.4$ & & $24,639.8$ \\
\hline & Mean & 0.7067 & $>$ & 0.6977 & $>$ & 0.7049 & $167,196.4$ & $>$ & $295,800.0$ & $>$ & $192,532.3$ & $196,833.3$ & $>$ & $322,600.0$ & $>$ & $228,033.3$ \\
\hline mitze & Stnd. & 0.004 & & 0.005 & & 0.004 & $71,342.0$ & & $120,451.6$ & & $85,651.1$ & $46,431.3$ & & $45,895.4$ & & $35,427.9$ \\
\hline & Mean & 0.7026 & $>$ & 0.6926 & $>$ & 0.7049 & $170,505.4$ & $>$ & $293,433.3$ & $>$ & $167,462.6$ & $138,746.6$ & $>$ & $238,966.7$ & $>$ & $150,369.3$ \\
\hline MIILLZ & Stnd. & 0.006 & & 0.005 & & 0.004 & $81,088.8$ & & $114,547.6$ & & $73,489.6$ & $43,605.3$ & & $31,957.4$ & & $26,484.5$ \\
\hline $\mathrm{J}$ & Mean & 0.7272 & $>$ & 0.7190 & $>$ & 0.7258 & $213,425.3$ & $>$ & $260,961.5$ & $>$ & $178,546.9$ & $129,838.5$ & $>$ & $218,833.3$ & $>$ & $144,924.0$ \\
\hline Mintzo & Stnd. & 0.00 & & 0.003 & & 0.004 & $110,600.2$ & & $98,614.1$ & & $83,908.9$ & $15,750.4$ & & $19,206.2$ & & $24,959.2$ \\
\hline & Mean & 0.7317 & $>$ & 0.7216 & $>$ & 0.7300 & $232,000.6$ & $>$ & $316,745.5$ & $>$ & $227,609.0$ & $106,494.9$ & $>$ & $156,466.7$ & $>$ & $123,967.4$ \\
\hline Instze & Stnd. & 0.004 & & 0.003 & & 0.004 & $89,860.0$ & & $109,571.5$ & & $108,245.1$ & $15,750.4$ & & $19,206.2$ & & $24,959.2$ \\
\hline
\end{tabular}


Table 12: Comparison of three MOEAs for two-objective supply chain problem in terms of different cost results which compose the total cost

\begin{tabular}{|l|l|c|c||c|c|c|c|c|c|}
\hline \multicolumn{2}{|l|}{ Inst. } & Total Risk & Total Cost & Service Level & Batch Cost & Production Cost & Transportation Cost & Stockout Costs & Holding Cost \\
\hline \multirow{2}{*}{ Inst2 } & NSGA-II & $17,220.8$ & $9,648.2$ & $98.24 \%$ & 450.0 & $6,571.8$ & 221.3 & $2,235.1$ & 170.0 \\
& SPEA2 & $15,023.0$ & $10,887.0$ & $98.44 \%$ & 400.0 & $7,616.1$ & 195.0 & $2,505.9$ & 170.0 \\
& IBEA & $15,282.9$ & $10,907.1$ & $98.44 \%$ & 420.0 & $7,544.9$ & 197.2 & $2,575.0$ & 170.0 \\
\hline \multirow{3}{*}{ Inst7 } & NSGA-II & $30,116.3$ & $21,677.5$ & $95.50 \%$ & 700.0 & $17,525.0$ & 443.0 & $2,034.5$ & 975.0 \\
& SPEA2 & $34,691.0$ & $24,153.5$ & $96.89 \%$ & 720.0 & $19,930.0$ & 454.0 & $2,299.5$ & 750.0 \\
& IBEA & $37,171.0$ & $22,166.5$ & $98.42 \%$ & 690.0 & $18,850.0$ & 405.0 & $1,871.5$ & 350.0 \\
\hline \multirow{2}{*}{ Inst13 } & NSGA-II & $57,172.0$ & $26,937.5$ & $98.61 \%$ & 900.0 & $21,570.0$ & 552.0 & $3,540.5$ & 375.0 \\
& SPEA2 & $57,558.4$ & $25,355.5$ & $98.03 \%$ & 910.0 & $20,365.0$ & 571.0 & $3,009.5$ & 500.0 \\
& IBEA & $58,166.6$ & $24,311.0$ & $98.15 \%$ & 850.0 & $19,400.0$ & 493.0 & $3,118.0$ & 450.0 \\
\hline \multirow{3}{*}{ Inst23 } & NSGA-II & $293,495.9$ & $86,074.0$ & $96.92 \%$ & $2,300.0$ & $64,535.2$ & $2,336.9$ & $14,540.7$ & $2,651.0$ \\
& SPEA2 & $301,443.0$ & $88,673.1$ & $96.12 \%$ & $2,310.0$ & $65,358.0$ & $2,210.0$ & $15,355.5$ & $3,439.0$ \\
& IBEA & $299,561.5$ & $88,115.6$ & $97.88 \%$ & $2,370.0$ & $66,971.1$ & $2,024.9$ & $14,878.6$ & $1,871.0$ \\
\hline
\end{tabular}

costs. For example, Table 12 shows that the SPEA2 solution is better than the IBEA solution in terms of both total cost and risk. However, service levels and holding costs are the same and more importantly, SPEA2 solution provides a better batch, transportation and stock out cost while IBEA solution provides a relatively better production cost in return worsening the cost for the remaining items. Considering Inst23, although in the overall, the IBEA solution is better (dominates) the SPEA2 solution, however again SPEA2 solution is better in terms of the production cost.

\section{Conclusions}

In this study, we addressed a supply chain management problem considering both supplier selection and inventory planning and used an Interval Type-2 Fuzzy System combined with an MOEA. We designed a two-stage approach for solving the problem; i) suppliers are ranked using IT2FSs, ii) supplier risk and operational costs for inventory planning are minimised using an MOEA. Hence, the proposed overall approach is capable of capturing the trade-off between risk and cost performing a search over the solution space accordingly and providing a set of 'equivalent' solutions. This gives decision makers the flexibility of choosing a solution from a set of trade-off solutions for supply chain management. We investigated the performance of the overall multi-objective approach using 
three well known MOEAs, namely NSGA-II, SPEA2 and IBEA for solving the integrated problem. Although, there are several studies on multi-objective Supply Chain Management (SCM)(Liao et al., 2011, Shankar et al. 2013, Zhang et al., 2016), to the authors' knowledge, this is one of the first studies in which the integrated problem of supplier selection and inventory planning has been investigated as a multi-objective problem.

Firstly, parameter settings of an MOEA does influence its performance and in most of the previous studies, parameter tuning appears to be a missing process (Liao et al., 2011, Shankar et al., 2013, Zhang et al., 2016). After parameter tuning, we used each MOEA at its best performance and tested them on twenty four problem instances. The empirical results indicate the overall success of NSGA-II interacting well with IT2FSs for SCM. All MOEAs achieved high quality trade-off solutions satisfying the customer demand almost fully in majority of the cases. A trivial future study could be applying the approach to new unseen instances possibly even larger than the ones used in this study and/or changing the decision makers' supplier related preferences creating more instances. We provide the problem instances used in this study as a benchmark along with our implementation of the approach for future research.

The empirical results indicate that there are even more conflicting objectives which can be considered in the solution model and then simultaneously optimised. Although MOEAs performed reasonably well in this study for the two-objective problem, this might not be the case when the number of objectives are increased. Recently, there has been a growing interest into many-objective (four or more objectives) optimisation considering that existing MOEAs could struggle in solving such problems (Deb \& Jain, 2014) requiring algorithmic improvement. For example, Deb \& Jain (2014) developed NSGA-III as an extension to NSGA-II with significant chances in the selection operator to overcome these difficulties. In future work, we intend to investigate the trade-off all contributing factors to the total cost and risk separately treating each as a separate objective as well as performances of many-objective approaches to those problems. 


\section{Acknowledgement}

The authors deeply grateful to Simon Miller for assistance with the model building code.

\section{References}

Altiparmak, F., Gen, M., Lin, L., \& Paksoy, T. (2006). A genetic algorithm approach for multi-objective optimization of supply chain networks. Computers E Industrial Engineering, 51, 196 - 215.

A.Sarkar, \& Mohapatra, P. (2006). Evaluation of supplier capability and performance: A method for supply base reduction. Journal of Purchasing and Supply Management, 12, 148 - 163.

Bechikh, S., Ben Said, L., \& Ghédira, K. (2010). Searching for knee regions in multi-objective optimization using mobile reference points. In Proceedings of the 2010 ACM Symposium on Applied Computing SAC '10 (pp. 1118-1125). New York, NY, USA: ACM.

Brockhoff, D., Friedrich, T., \& Neumann, F. (2008). Analyzing Hypervolume Indicator Based Algorithms. In G. Rudolph et al. (Eds.), Conference on Parallel Problem Solving From Nature (PPSN X) (pp. 651-660). Springer volume 5199 of $L N C S$.

Chen, C.-T., Lin, C.-T., \& Huang, S.-F. (2006). A fuzzy approach for supplier evaluation and selection in supply chain management. International Journal of Production Economics, 102, 289 - 301.

Coello, C. A. C., Lamont, G. B., \& Veldhuizen, D. A. V. (2006). Evolutionary Algorithms for Solving Multi-Objective Problems (Genetic and Evolutionary Computation). Secaucus, NJ, USA: Springer-Verlag New York, Inc.

Coello Coello, C., \& Reyes Sierra, M. (2004). A study of the parallelization of a coevolutionary multi-objective evolutionary algorithm. In R. Monroy, 
G. Arroyo-Figueroa, L. Sucar, \& H. Sossa (Eds.), MICAI 2004: Advances in Artificial Intelligence (pp. 688-697). Springer Berlin Heidelberg volume 2972 of Lecture Notes in Computer Science.

Czyzak, P., \& Jaszkiewicz, A. (1998). Pareto simulated annealing - a metaheuristic technique for multiple-objective combinatorial optimization. Journal of Multi-Criteria Decision Analysis, 7, 34-47.

Deb, K. (2007). Evolutionary multi-objective optimization without additional parameters. In F. G. Lobo, C. F. Lima, \& Z. Michalewicz (Eds.), Parameter Setting in Evolutionary Algorithms (pp. 241-257). Berlin, Heidelberg: Springer Berlin Heidelberg.

Deb, K., \& Jain, H. (2014). An evolutionary many-objective optimization algorithm using reference-point-based nondominated sorting approach, part i: Solving problems with box constraints. IEEE Transactions on Evolutionary Computation, 18, 577-601.

Deb, K., Pratap, A., Agarwal, S., \& Meyarivan, T. (2002). A fast and elitist multiobjective genetic algorithm: Nsga-ii. Evolutionary Computation, IEEE Transactions on, 6, 182-197.

Durillo, J., Nebro, A., \& Alba, E. (2010). The jmetal framework for multiobjective optimization: Design and architecture. In CEC 2010 (pp. 41384325). Barcelona, Spain.

Durillo, J. J., \& Nebro, A. J. (2011). jmetal: A java framework for multiobjective optimization. Advances in Engineering Software, 42, 760-771.

Esmin, A. A., Coelho, R. A., \& Matwin, S. (2015). A review on particle swarm optimization algorithm and its variants to clustering high-dimensional data. Artif. Intell. Rev., 44, 23-45.

Ghodsypour, S., \& Brien, C. O. (2001). The total cost of logistics in supplier selection, under conditions of multiple sourcing, multiple criteria and capacity 
constraint. International Journal of Production Economics, 73, 15 - 27. Supply Chain Management.

Gong, Y. (2013). Fuzzy multi-attribute group decision making method based on interval type-2 fuzzy sets and applications to global supplier selection. International Journal of Fuzzy Systems, 15.

Greenfield, S., Chiclana, F., John, R., \& Coupland, S. (2012). The sampling method of defuzzification for type-2 fuzzy sets: Experimental evaluation. Information Sciences, 189, $77-92$.

Ho, W., Xu, X., \& Dey, P. K. (2010). Multi-criteria decision making approaches for supplier evaluation and selection: A literature review. Eur. J. Oper. Res., 202, $16-24$.

Hu, J., Zhang, Y., Chen, X., \& Liu, Y. (2013). Multi-criteria decision making method based on possibility degree of interval type-2 fuzzy number. Knowledge-Based Systems, 43, 21-29.

Kim, I., \& de Weck, O. (2006). Adaptive weighted sum method for multiobjective optimization: a new method for pareto front generation. Structural and Multidisciplinary Optimization, 31, 105-116.

Konak, A., Coit, D. W., \& Smith, A. E. (2006). Multi-objective optimization using genetic algorithms: A tutorial. Reliability Engineering and System Safety, 91, 992 - 1007. Special Issue - Genetic Algorithms and ReliabilitySpecial Issue - Genetic Algorithms and Reliability.

Liao, S.-H., Hsieh, C.-L., \& Lai, P.-J. (2011). An evolutionary approach for multi-objective optimization of the integrated locationâ $€$ "inventory distribution network problem in vendor-managed inventory. Expert Systems with Applications, 38, $6768-6776$.

Mahnam, M., Yadollahpour, M. R., Famil-Dardashti, V., \& Hejazi, S. R. (2009). Supply chain modeling in uncertain environment with bi-objective approach. Computers $\&$ Industrial Engineering, 56, 1535 - 1544. 
Mastrocinque, E., Yuce, B., Lambiase, A., \& Packianather, M. (2013). A multiobjective optimization for supply chain network using the bees algorithm. International Journal of Engineering Business Management, 5, 1-11.

Mendel, J. M., John, R., \& Liu, F. (2006). Interval type-2 fuzzy logic systems made simple. IEEE T. Fuzzy Systems, 14, 808-821.

Mendel, J. M., \& John, R. B. (2002). Type-2 fuzzy sets made simple. Fuzzy Systems, IEEE Transactions on, 10, 117-127.

Miller, S., Gongora, M., Garibaldi, J., \& John, R. (2012). Interval type-2 fuzzy modelling and stochastic search for real-world inventory management. Soft Computing, 16, 1447-1459.

Miller, S., Gongora, M., \& John, R. (2011). Interval type-2 fuzzy modelling and simulated annealing for real-world inventory management. In Hybrid Artificial Intelligence Systems 2011 (HAIS2011) 23-25 May, 2011 Wroclaw, Poland (pp. 231-238).

Miller, S., \& John, R. (2010). An interval type-2 fuzzy multiple echelon supply chain model. Knowledge-Based Systems, 23, 363 - 368. Artificial Intelligence 2009 AI-2009 The 29th \{SGAI\} International Conference on Artificial Intelligence.

Mohammaditabar, D., \& Ghodsypour, S. H. (2014). A supplier-selection model with classification and joint replenishment of inventory items. International Journal of Systems Science, 0, 1-10.

Narukawa, K., \& Rodemann, T. (2012). Examining the performance of evolutionary many-objective optimization algorithms on a real-world application. In Proceedings of the 2012 Sixth International Conference on Genetic and Evolutionary Computing ICGEC '12 (pp. 316-319). Washington, DC, USA: IEEE Computer Society.

Ordoobadi, S. M. (2009). Development of a supplier selection model using fuzzy logic. Supply Chain Management: An International Journal, 14, 314-327. 
Parhizkari, M., Amiri, M., \& Mousakhani, M. (2013). A multiple criteria decision making technique for supplier selection and inventory management strategy: A case of multi-product and multi-supplier problem. Decision Science Letters, 2, $185-190$.

Pattnaik, M. (2011). Supplier selection strategies on fuzzy decision space. General Mathematics Notes, 4, $49-69$.

Rezaei, J., \& Davoodi, M. (2008). A deterministic, multi-item inventory model with supplier selection and imperfect quality. Applied Mathematical Modelling, 32, $2106-2116$.

Ross, T. J. (2004). Fuzzy Logic with Engineering Applications. John Wiley \& Sons.

Sadeghi, J., Sadeghi, S., \& Niaki, S. T. A. (2014). A hybrid vendor managed inventory and redundancy allocation optimization problem in supply chain management: An nsga-ii with tuned parameters. Computers 83 Operations Research, 41, $53-64$.

Sarker, R., \& Coello Coello, C. (2002). Assessment methodologies for multiobjective evolutionary algorithms. In Evolutionary Optimization (pp. 177-195). Springer US volume 48 of International Series in Operations Research $\& 3$ Management Science.

Setak, M., Sharifi, S., \& madian, A. A. (2012). Supplier selection and order allocation models in supply chain management: a review. World Applied Sciences Journal, 18, $55-72$.

Shankar, B. L., Basavarajappa, S., Kadadevaramath, R. S., \& Chen, J. C. (2013). A bi-objective optimization of supply chain design and distribution operations using non-dominated sorting algorithm: A case study. Expert Systems with Applications, 40, $5730-5739$. 
Sierra, M. R., \& Coello, C. A. C. (2004). A new multi-objective particle swarm optimizer with improved selection and diversity mechanisms. In Proceeding of the 2004 Congress on Evolutionary Computation (pp. 1-39).

Taguchi, G., \& Yokoyama, Y. (1993). Taguchi methods: design of experiments. TAGUCHI METHODS SERIES. ASI Press.

Thomas, D. J., \& Griffin, P. M. (1996). Coordinated supply chain management. Eur. J. Oper. Res., 94, $1-15$.

Turk, S., John, R., \& Özcan, E. (2014). Interval type-2 fuzzy sets in supplier selection. In 14th UK Workshop on Computational Intelligence UKCI2014 (pp. 1-7).

Turk, S., Miller, S., Özcan, E., \& John, R. I. (2015). A simulated annealing approach to supplier selection aware inventory planning. In IEEE Congress on Evolutionary Computation, CEC 2015, Sendai, Japan, May 25-28, 2015 (pp. 1799-1806).

Veldhuizen, D. A. V., \& Veldhuizen, D. A. V. (1999). Multiobjective Evolutionary Algorithms: Classifications, Analyses, and New Innovations. Technical Report Evolutionary Computation.

Vonderembse, M. A., \& Tracey, M. (1999). The impact of supplier selection criteria and supplier involvement on manufacturing performance. Journal of Supply Chain Management, 35, 33-39.

Zadeh, L. (1965). Fuzzy sets. Information and Control, 8, $338-353$.

Zhang, S., Lee, C. K. M., Wu, K., \& Choy, K. L. (2016). Multi-objective optimization for sustainable supply chain network design considering multiple distribution channels. Expert Systems with Applications, 65, 87 - 99.

Zitzler, E. (1999). Evolutionary algorithms for multiobjective optimization: Methods and applications. 
Zitzler, E., Brockhoff, D., \& Thiele, L. (2007). The hypervolume indicator revisited: On the design of pareto-compliant indicators via weighted integration. In S. Obayashi, K. Deb, C. Poloni, T. Hiroyasu, \& T. Murata (Eds.), Evolutionary Multi-Criterion Optimization (pp. 862-876). Springer Berlin Heidelberg volume 4403 of Lecture Notes in Computer Science.

Zitzler, E., Deb, K., \& Thiele, L. (2000). Comparison of multiobjective evolutionary algorithms: Empirical results. Evol. Comput., 8, 173-195.

Zitzler, E., \& Künzli, S. (2004). Indicator-Based Selection in Multiobjective Search. In X. Yao et al. (Eds.), Conference on Parallel Problem Solving from Nature (PPSN VIII) (pp. 832-842). Springer volume 3242 of LNCS.

Zitzler, E., Laumanns, M., \& Thiele, L. (2002). Spea2: Improving the strength pareto evolutionary algorithm for multiobjective optimization. In Evolutionary Methods for Design, Optimisation, and Control (pp. 95-100). CIMNE, Barcelona, Spain.

Zitzler, E., \& Thiele, L. (1999). Multiobjective evolutionary algorithms: a comparative case study and the strength pareto approach. Evolutionary Computation, IEEE Transactions on, 3, 257-271. 